\title{
Potential inhibitors against papain-like protease of novel coronavirus (COVID-19) from FDA approved drugs
}

\author{
Rimanshee Arya ${ }^{1}$, Amit Das', Vishal Prashar ${ }^{1, *}$, Mukesh Kumar ${ }^{1,2, *}$ \\ ${ }^{1}$ Radiation Biology \& Health Sciences Division, Bhabha Atomic Research Centre, Trombay, \\ Mumbai-400085, India \\ ${ }^{2}$ Homi Bhabha National Institute, Mumbai-400094, India
}

*Correspondence should be addressed to VP (Email:vishalp@,barc.gov.in) and MK (Email: mukeshk@barc.gov.in)

\begin{abstract}
The cases of 2019 novel coronavirus (COVID-19) infection have been continuously increasing ever since its outbreak in China last December. Currently, there are no approved drugs to treat the infection. In this scenario, there is a need to utilize the existing repertoire of FDA approved drugs to treat the disease. The rational selection of these drugs could be made by testing their ability to inhibit any COVID-19 proteins essential for viral life-cycle. We chose one such crucial viral protein, the papain-like protease (PLpro), to screen the FDA approved drugs in silico. The homology model of the protease was built based on the SARS-coronavirus PLpro structure, and the drugs were docked in S3/S4 pockets of the active site of the enzyme. In our docking studies, fifteen FDA approved drugs, including chloroquine and formoterol, bind the target enzyme with significant affinity and good geometry, suggesting their potential to be utilized against the virus.
\end{abstract}

\section{Introduction}

The recent outbreak of 2019 novel coronavirus (COVID-19) in China has been the cause of major concern for the global community as the number of people infected with the virus has been continuously increasing with a significant geographical spread. As on $15^{\text {th }}$ February 2020, WHO reports 50,580 confirmed cases of infection globally with 1526 deaths. Several attempts are being made to find new therapeutics against COVID-19. China's National Health Commission has recommended using HIV-1 protease inhibitors, lopinavir, and ritonavir as an ad hoc treatment against the infection while Wang et al. have tested seven approved drugs in vitro against the clinical isolate of the virus (Wang et al., 2020). The robust research on other aspects of COVID19, including epidemiology and genome sequencing, has provided useful insights into the new virus (Lu et al, 2020; Chan et al, 2020). The complete genome sequencing showed that the virus 
belonged to a large family of coronaviruses and is closely related to Severe Acute Respiratory Syndrome-Coronavirus (SARS-CoV). It encodes for two large polyproteins that are further processed by virally encoded cysteine proteases, namely, the papain-like protease (PLpro) and the 3-chymotrypsin-like protease (3CLpro, also known as the main protease-Mpro). The processing of the viral polyproteins is essential for maturation and infectivity of the virus (Chen et al., 2020). Because of the crucial roles these proteases play in the viral life-cycle, they are important targets for antiviral drug design.

Recently, COVID-19 Mpro has been used as a target to screen clinically approved drugs as potential inhibitors (Xu et al., 2020; Liu et al., 2020; Li et al., 2020). Since the safety profile of these FDA approved drugs is well documented and the efficacy of selected few can be quickly tested against a viral culture, the drug repurposing could be an efficient approach to find therapeutics against COVID-19. We have carried out the virtual screening of these drugs against COVID-19 PLpro to find potential inhibitors of its catalytic domain.

\section{Materials and Methods}

\section{Homology modeling}

The protein sequence of COVID-19 PLpro was obtained from GenBank (Accession QHD 43415). The catalytic domain of PLpro was delineated by comparing it with the known sequences of other coronaviruses. The homology model of the COVID-19 PLpro catalytic domain was generated in the SWISS-MODEL workspace ( CoV PLpro crystal structure (PDB Id: 3E9S) as a template.

\section{Virtual screening}

A total of 2525 FDA approved drugs used for docking studies were downloaded either from the DrugBank database (www.drugbank.ca) or the Zinc15 library (Sterling and Irwin, 2015). Docking was performed using the SEESAR suite of programs from BioSolveIT (www.biosolveit.de/SeeSAR).

\section{Results and Discussion}

The COVID-19 PLpro is responsible for processing three cleavage sites of the viral polyprotein to release mature non-structural proteins 1, 2 and 3. Apart from proteolytic processing, PLpro also has a deubiquitinase and deISGylating activity. There were several earlier attempts to design inhibitors 
against SARS-CoV PLpro and they yielded promising results (Ratia et al., 2008; Ghosh et al., 2009, 2010; Baez-Santos et al., 2014). We, therefore, chose COVID-19 PLpro to virtually screen FDA approved drugs to find potential therapeutics. The multiple sequence alignment of the catalytic domain of PLpro from COVID-19 with other coronaviruses shows their close similarity as shown in Figure 1.

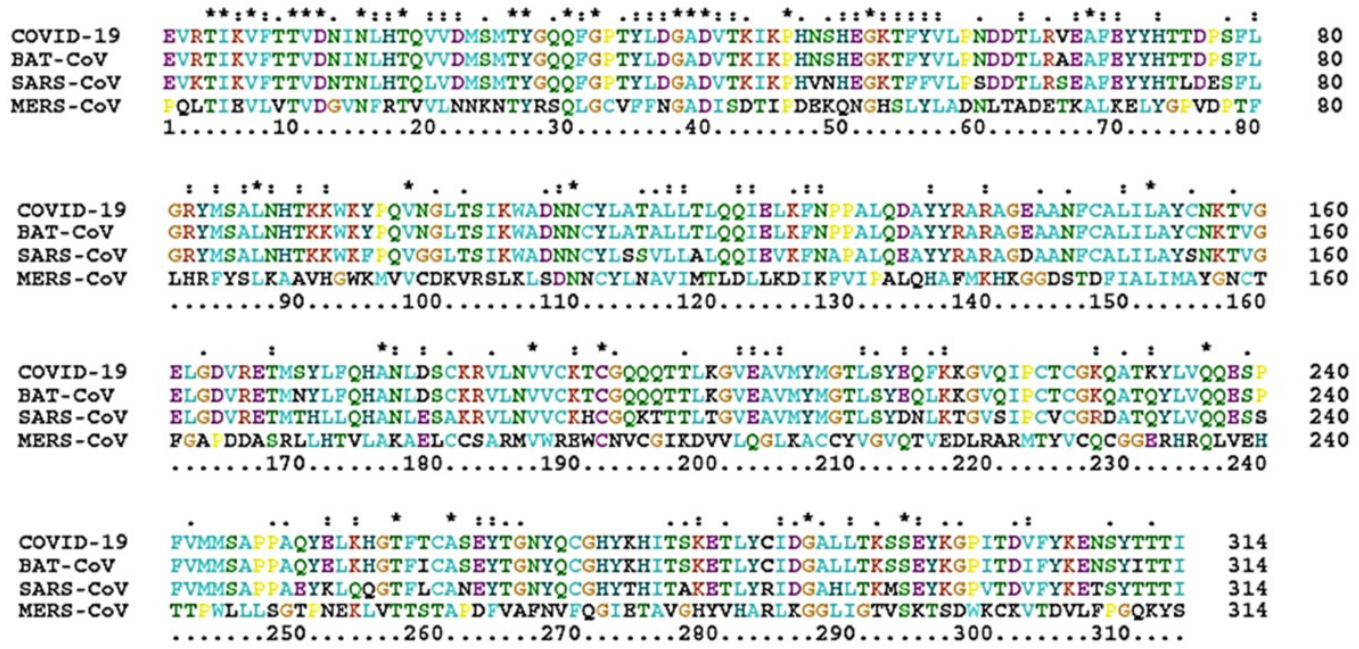

Figure 1: Multiple sequence alignment of the catalytic domains of papain-like proteases (PLpro) from different coronaviruses.

The homology model of COVID-19 PLpro was generated using the SARS-CoV PLpro crystal structure (PDB Id: 3E9S) as a template. The homology model was in a closed flap conformation with GMQE and QMean scores (Benkert et al., 2008) of 0.95 and -0.22 , respectively. The homology model could accommodate the ligand from the template well. Therefore, the template ligand (an inhibitor of SARS-CoV PLpro) was used to define the binding site for docking. The binding site contained more spacious $\mathrm{S} 3 / \mathrm{S} 4$ pockets, rather than the restrictive $\mathrm{S} 1 / \mathrm{S} 2$ pockets close to the catalytic residues. The S3/S4 pocket contained residues Asp164, Val165, Arg166, Glu167, Met 208, Ala246, Pro247, Pro248, Tyr 264, Gly266, Asn267, Tyr 268, Gln269, Cys217, Gly271, Tyr273, Thr301 and Asp302. For each ligand, ten poses were generated and docked into the binding site. All these poses were sorted according to their binding affinities. Thirty-four drugs had binding affinity better than $100 \mu \mathrm{M}$ with no torsional strain, intra- and inter-molecular clashes. These compounds were then inspected visually. Out of thirty-four drugs, the ligand efficiency (LE) value was +2 for ten drugs, +1 for fourteen drugs, and 0 for seven drugs. The remaining drugs had negative LE values and were discarded from further consideration. Among the selected set, there were several anesthetics, 
antineoplastic agents, appetite depressants, skin ointments, diagnostic imaging agents and other unsuitable drugs, and hence were removed from consideration. Finally, fifteen drugs were left with an estimated binding affinity within $8 \mathrm{nM}$ to $100 \mu \mathrm{M}$ range, having no torsional strain, intra- and inter-molecular clashes. These promising inhibitors of COVID-19 PLpro are listed in Table 1, and their docked poses are shown in Figure 2.

Table 1: Fifteen FDA approved drugs showing the best affinity to COVID-19 PLpro

\begin{tabular}{|c|c|c|c|}
\hline Name & Binding Affinity & L.E. & Current Application \\
\hline 1. Biltricide & $8 \mathrm{nM}-8 \mu \mathrm{M}$ & + & Anthelmintic \\
\hline 2. Cinacalcet & $26 \mathrm{nM}-3 \mu \mathrm{M}$ & 0 & $\begin{array}{l}\text { Calcimimetic, to treat } \\
\text { hyperparathyroidism }\end{array}$ \\
\hline 3. Procainamide & $30 \mathrm{nM}-3 \mu \mathrm{M}$ & ++ & Antiarrhythmic \\
\hline 4. Terbinafine & $33 \mathrm{nM}-3 \mu \mathrm{M}$ & + & Anti-fungal \\
\hline 5. Pethidine & $53 \mathrm{nM}-5 \mu \mathrm{M}$ & + & Narcotic analgesic \\
\hline 6. Labetalol & $113 \mathrm{nM}-11 \mu \mathrm{M}$ & 0 & To treat hypertension \\
\hline 7. Tetrahydrozoline & $137 \mathrm{nM}-14 \mu \mathrm{M}$ & ++ & $\begin{array}{l}\text { Over the counter eye drops and nasal } \\
\text { spray }\end{array}$ \\
\hline 8. Ticlopidine & $160 \mathrm{nM}-16 \mu \mathrm{M}$ & + & Inhibitor of platelet aggregation. \\
\hline 9. Ethoheptazine & $163 \mathrm{nM}-16 \mu \mathrm{M}$ & + & Opioid analgesic \\
\hline 10.Levamisole & $259 \mathrm{nM}-26 \mu \mathrm{M}$ & ++ & $\begin{array}{l}\text { Antihelminthic used for parasitic, viral, } \\
\text { and bacterial infections. }\end{array}$ \\
\hline 11.Amitriptyline & $466 \mathrm{nM}-46 \mu \mathrm{M}$ & 0 & antidepressant with analgesic properties \\
\hline 12.Naphazoline & $697 \mathrm{nM}-69 \mu \mathrm{M}$ & + & $\begin{array}{l}\text { Decongestant in over-the-counter eye } \\
\text { drops and nasal preparations. }\end{array}$ \\
\hline 13.Formoterol & $716 \mathrm{nM}-71 \mu \mathrm{M}$ & 0 & Management of COPD and asthma. \\
\hline 14.Chloroquine & $858 \mathrm{nM}-85 \mu \mathrm{M}$ & 0 & Antimalarial agent \\
\hline 15.Chlorothiazide & $939 \mathrm{nM}-93 \mu \mathrm{M}$ & + & Diuretic \\
\hline
\end{tabular}



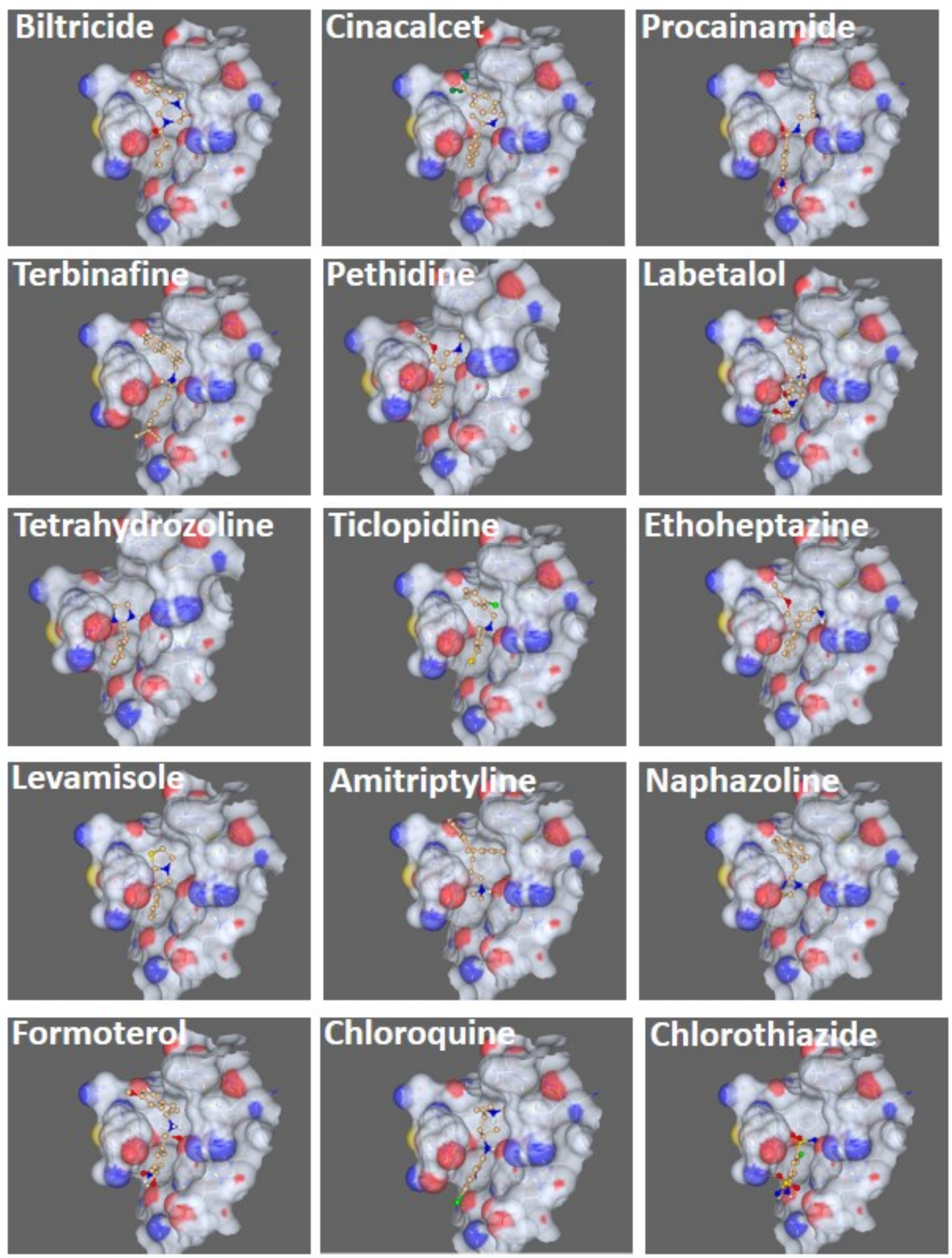

Figure 2: Docked poses of selected FDA approved drugs in the S3/S4 binding site of COVID19 PLpro. The drugs are shown as ball-and-stick models inside the binding site (shown as semitransparent protein surface). The figures were drawn in Seesar (www.biosolveit.de/SeeSAR). 
Interestingly, our analysis picked up the anti-malarial drug, chloroquine, as a potential inhibitor of viral PLpro. The anti-viral activity of chloroquine has been reported earlier (Savarino et al., 2006 \& Yan et al., 2013). Recently, China has launched a few trials of the drug in patients after it was shown to block the COVID-19 infection at a low micro-molar concentration $\left(\mathrm{EC}_{50}: 1.13 \mu \mathrm{M}\right)$ in cell culture experiment (Wang et al., 2020). It was found that chloroquine works against the viral infection at the entry-level, as well as post-entry stages. It is likely that the effect of chloroquine during postentry stages might be manifested through its inhibition of the crucial viral protein, PLpro.

The other interesting molecule picked up in our analysis is formoterol, which relaxes muscles in the airways to improve breathing and is used as a bronchodilator in the management of chronic obstructive pulmonary disease (COPD) and asthma. The drug will have a synergistic effect in treating patients if it also inhibited the activity of viral PLpro.

While the search for new therapeutics against COVID-19 continues, it will take time for a novel molecule to reach clinics. Therefore, exploring the potential of existing drugs in a rational way assumes significance. Our in silico study shows that fifteen FDA approved drugs have the potential to inhibit COVID-19 PLpro, and they should be evaluated in virus cultures to assess their effectiveness.

\section{References}

Báez-Santos, Yahira M., Scott J. Barraza, Michael W. Wilson, Michael P. Agius, Anna M. Mielech, Nicole M. Davis, Susan C. Baker, Scott D. Larsen, and Andrew D. Mesecar. (2014) $\mathrm{X}$-ray structural and biological evaluation of a series of potent and highly selective inhibitors of human coronavirus papain-like proteases. J Med Chem, 57, 2393-2412.

Benkert P, Tosatto SC, Schomburg D. (2008) QMEAN: A comprehensive scoring function for model quality assessment. Proteins, 71, 261-77.

Chan, Jasper Fuk-Woo, Shuofeng Yuan, Kin-Hang Kok, Kelvin Kai-Wang To, Hin Chu, Jin Yang, Fanfan Xing et al. (2020) A familial cluster of pneumonia associated with the 2019 novel coronavirus indicating person-to-person transmission: a study of a family cluster. Lancet, https://doi.org/10.1016/S0140-6736(20)30154-9

Chen, Yu, Qianyun Liu, and Deyin Guo. (2020) Coronaviruses: genome structure, replication, and pathogenesis. J Med Virology, https://doi.org/10.1002/jmv.25681 
Ghosh, Arun K., Jun Takayama, Yoann Aubin, Kiira Ratia, Rima Chaudhuri, Yahira Baez, Katrina Sleeman et al. (2009) Structure-Based Design, Synthesis, and Biological Evaluation of a Series of Novel and Reversible Inhibitors for the Severe Acute Respiratory SyndromeCoronavirus Papain-Like Protease. J Med Chem, 52, 5228-5240.

Ghosh, Arun K., Jun Takayama, Kalapala Venkateswara Rao, Kiira Ratia, Rima Chaudhuri, Debbie C. Mulhearn, Hyun Lee et al. (2010) Severe acute respiratory syndrome coronavirus papain-like novel protease inhibitors: design, synthesis, protein-ligandd X-ray structure and biological evaluation. J Med Chem, 53, 4968-4979.

Liu, Xin, and Xiu-Jie Wang. "Potential inhibitors for 2019-nCoV coronavirus M protease from clinically approved medicines." bioRxiv (2020) https://doi.org/10.1101/2020.01.29.924100

Li, Yan, Jinyong Zhang, Ning Wang, Haibo Li, Yun Shi, Gang Guo, Kaiyun Liu, Hao Zeng, and Quanming Zou. (2020) Therapeutic Drugs Targeting 2019-nCoV Main Protease by HighThroughput Screening. bioRxiv, https://doi.org/10.1101/2020.01.28.922922

Lu, Roujian, Xiang Zhao, Juan Li, Peihua Niu, Bo Yang, Honglong Wu, Wenling Wang et al. (2020) Genomic characterisation and epidemiology of 2019 novel coronavirus: implications for virus origins and receptor binding. Lancet, https://doi.org/10.1016/S0140-6736(20)30251-8

Ratia, Kiira, Scott Pegan, Jun Takayama, Katrina Sleeman, Melissa Coughlin, Surendranath Baliji, Rima Chaudhuri et al. (2008) A noncovalent class of papain-like protease/deubiquitinase inhibitors blocks SARS virus replication. Proc Nat Acad Sci (USA), 105, 16119-16124.

Savarino, A., Di Trani, L., Donatelli, I., Cauda, R. \& Cassone, A. (2006) New insights into the antiviral effects of chloroquine, Lancet Infect Dis, 6, 67-69

SeeSAR version 9.2; BioSolveIT GmbH, Sankt Augustin, Germany, (2019), $\underline{\text { www.biosolveit.de/SeeSAR }}$

Sterling, T. and Irwin, J. J. (2015) ZINC 15 - Ligand Discovery for Everyone. J Chem Inf Model, 55, 2324-2337.

Wang, M., Cao, R., Zhang, L. et al. (2020) Remdesivir and chloroquine effectively inhibit the recently emerged novel coronavirus $(2019-\mathrm{nCoV})$ in vitro. Cell Res, https://doi.org/10.1038/s41422-020-0282-0. 
Xu, Zhijian, Cheng Peng, Yulong Shi, Zhengdan Zhu, Kaijie Mu, Xiaoyu Wang, and Weiliang Zhu. (2020) Nelfinavir was predicted to be a potential inhibitor of 2019-nCov main protease by an integrative approach combining homology modelling, molecular docking and binding free energy calculation. bioRxiv, https://doi.org/10.1101/2020.01.27.921627

Yan, Y., Zou, Z., Sun, Y., Li, X., Xu, K.F., Wei, Y., Jin, N., Jiang, C. (2013) Anti-malaria drug chloroquine is highly effective in treating avian influenza A H5N1 virus infection in an animal model, Cell Res. 23, 300-302. 